\title{
A New Type of Computable Inductor
}

\section{Chester H. Page}

\author{
(November 21, 1962)
}

The mutual inductance analog of the generalized Thompson-Lampard theorem (for cross capacitances) is developed. An infinitely long cage of five parallel wires can yield an absolute inductance of

$$
10^{-7} \ln \frac{3+\sqrt{5}}{2}
$$

\begin{abstract}
henries per meter. End-effects of order $1 / l^{2}$ occur in a finite cage, but can be reduced to order $1 / l^{4}$ by using eight wires.

The eight-wire cage has the advantage of overdetermined relations among the inductances to be measured, allowing an estimate of experimental error in the calibration of a standard. Errors due to faulty eage geometry are shown to be of the order of 1 in $10^{7}$.
\end{abstract}

The Thompson-Lampard theorem relates to the cross-capacitances of a cylindrical configuration of conductors. Consider a conducting cylindrical shell whose right cross section is an arbitrary closed curve, divided into four segments by infinitesimal gaps. The generalized theorem ${ }^{1}$ states that the two cross capacitances, per unit length of the cylinder, are related by

$$
e^{-4 \pi^{2} C_{1} / \Gamma_{e}}+e^{-4 \pi^{2} C_{2} / \Gamma_{e}}=1
$$

where $\Gamma_{e}$ (often written $\epsilon_{0}$ ) is the electric constant of the system of units considered.

The generality of this theorem suggests that an analogous theorem should exist for the mutual inductances of a cage of parallel wires. Consider first an infinitely long cage of four parallel wires, penetrating a transverse plane as in figure 1.

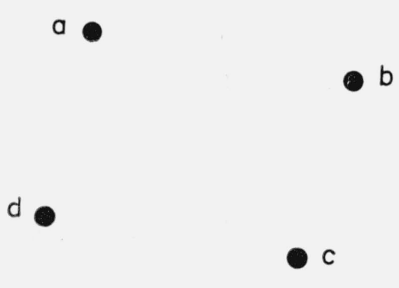

Figure 1

Three mutual inductances are involved; the pairs of loops whose traces in the plane are (1) $\overline{a b}$ and $\overline{c d},(2) \overline{a d}$ and $\overline{b c},(3) \overline{a c}$ and $\overline{b d}$. In case (1), the mutual inductance per unit cage length is

$$
m=\frac{\Gamma_{m}}{2 \pi} \ln \frac{\overline{a c} \overline{b d}}{\overline{a d}} \overline{b c}
$$

where $\Gamma_{m}$ is the magnetic constant, often written $\mu_{0}$. By considering $a, b, c, d$ to be points in a complex variable plane, we can write

$$
m=\frac{\Gamma_{m}}{2 \pi} \ln \left|\frac{(a-c)(b-d)}{(a-d)(b-c)}\right| .
$$

1 D. G. Lampard and R. D. Cutkosky, Some results on the cross capacitances per unit length of cylindrical three-terminal capacitors with thin dielectric films on their electrodes, I.E.E. Monograph $351 \mathrm{M}, 1960$. 
We shall find it convenient to use

$$
m=-\frac{\Gamma_{m}}{2 \pi} \ln |x|=-\frac{\Gamma_{m}}{4 \pi} \ln A
$$

where $A$ is the square of the absolute value of the cross ratio $x$.

The desired theorem is a nonhomogeneous identity among the several mutual inductances. It can be expressed as an identity among the cross ratios. Thus the problem is to find a geometrical identity among the cross ratios of a set of points in a plane.

Now

$$
x_{1} \equiv \frac{(a-d)(b-c)}{(a-c)(b-d)} \quad x_{2} \equiv \frac{(a-b)(d-c)}{(a-c)(d-b)}
$$

yielding the identity

$$
x_{1}+x_{2} \equiv 1 \text {. }
$$

If the four points lie on a circle (including a straight line), the cross ratios are real (and positive), so that $\left|x_{1}\right|+\left|x_{2}\right|=1$, yielding

$$
e^{-2 \pi m_{1} / \Gamma_{m}}+e^{-2 \pi m_{2} / \Gamma_{m}}=1
$$

corresponding to the Thompson-Lampard theorem. Unfortunately, departures from a circular locus make the $x$ 's complex, and eq (4) no longer implies any relation between the $|x|$ 's.

The third possible pairing corresponds to crossed loops, with

$$
x_{3}=\frac{(a-b)(c-d)}{(a-d)(c-b)}
$$

and is related to the others through

$$
x_{\mathbf{3}} \equiv-x_{2} / x_{1}
$$

yielding the homogeneous identity,

$$
m_{3}=\left|m_{2}-m_{1}\right|
$$

which is a circuit relation, independent of the cage configuration:

\section{Pentagonal Cage}

For a five-wire cage, there are five pairs of sides (fig. 2).

\section{$z_{4}$}
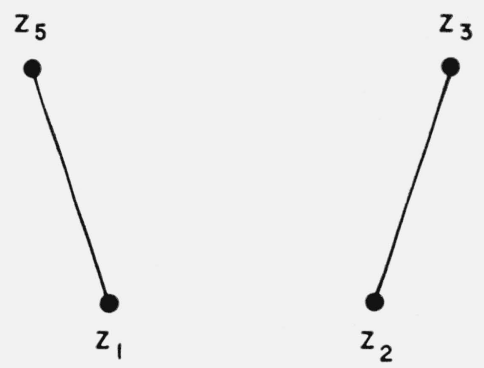

Figure 2

(Illustrating loops for $M_{1}$ ). 
There are five mutual inductances corresponding to pairs of nonadjacent sides:

$$
x_{i} \equiv \frac{\left(z_{i+1}-z_{i}\right)\left(z_{i+2}-z_{i-1}\right)}{\left(z_{i+1}-z_{i-1}\right)\left(z_{i+2}-z_{i}\right)}, \text { indices modulo } 5
$$

There are also five crossed-pairs (fig. 3):

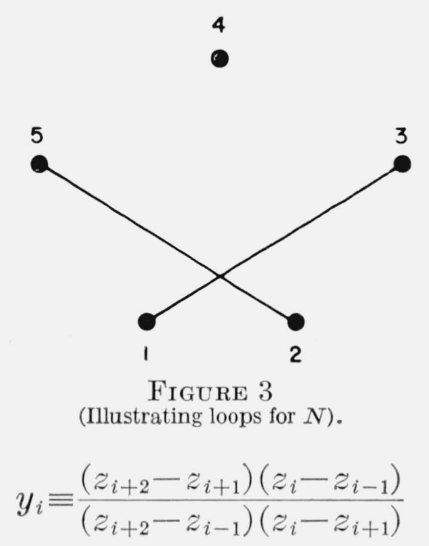

related to the $x_{i}$ through the identity

$$
x_{i} y_{i} \equiv-x_{i-1} x_{i+1}
$$

which yields the circuit relation

$$
m_{i}+n_{i}=m_{i-1}+m_{i+1} .
$$

The five $x_{i}$ are mutually related by the nonhomogeneous identities

$$
x_{i}+x_{i-1} x_{i+1} \equiv 1 \text {. }
$$

These identities will be shown to yield an identity among $\left|x_{i}\right|$, and therefore among the $m_{i}$. The result is the desired analog of the Thompson-Lampard theorem.

\section{Derivation of Theorem for Pentagonal Cage}

The identities (6) with $i=1$ and $i=2$ can be combined to yield

$$
x_{3}+x_{5}-\frac{1}{x_{1}}-\frac{1}{x_{2}}=-\frac{x_{1}}{x_{2}}-\frac{x_{2}}{x_{1}}
$$

The identities (6) for $i=1,2,3,5$ yield directly

$$
\begin{aligned}
& \left|1-x_{3}\right|^{2}=\left|x_{2} x_{4}\right|^{2}=A_{2} A_{4} \\
& \left|1-x_{5}\right|^{2}=\left|x_{1} x_{4}\right|^{2}=A_{1} A_{4} \\
& \left|1-\frac{1}{x_{1}}\right|^{2}=\left|\frac{x_{2} x_{5}}{x_{1}}\right|^{2}=A_{2} A_{5} / A_{1} \\
& \left|1-\frac{1}{x_{2}}\right|^{2}=\left|\frac{x_{1} x_{3}}{x_{2}}\right|^{2}=A_{1} A_{3} / A_{2}
\end{aligned}
$$

where

$$
A_{i} \equiv\left|x_{i}\right|^{2} .
$$


We also have the purely algebraic identity

$$
\left|1-x_{3}\right|^{2}+\left|1-x_{5}\right|^{2}-\left|1-\frac{1}{x_{1}}\right|^{2}-\left|1-\frac{1}{x_{2}}\right|^{2}-\left|x_{3}\right|^{2}-\left|x_{5}\right|^{2}+\left|\frac{1}{x_{1}}\right|^{2}+\left|\frac{1}{x^{2}}\right|^{2} \equiv-2 \operatorname{Re}\left(x_{3}+x_{5}-\frac{1}{x_{1}}-\frac{1}{x_{2}}\right) .
$$

Using (7) and (8), this becomes

$$
\begin{aligned}
A_{2} A_{4}+A_{1} A_{4}-A_{2} A_{5} / A_{1}-A_{1} A_{3} / A_{2}-A_{3}-A_{5}+1 / A_{1}+1 / A_{2} & =2 \operatorname{Re}\left(\frac{x_{1}}{x_{2}}+\frac{x_{2}}{x_{1}}\right) \\
& =2 \operatorname{Re}\left(\frac{x_{1} \overline{x_{2}}}{A_{2}}+\frac{x_{2} \overline{x_{1}}}{A_{1}}\right) \\
& =2\left(\alpha_{1} \alpha_{2}+\beta_{1} \beta_{2}\right)\left(\frac{1}{A_{1}}+\frac{1}{A_{2}}\right)
\end{aligned}
$$

where

$$
x_{i} \equiv \alpha_{i}+j \beta_{i}, \quad \overline{x_{i}} \equiv \alpha_{i}-j \beta_{i} .
$$

Now identity (6) for $i=1, \quad x_{1}+x_{2} x_{5} \equiv 1$

yields

$$
\begin{aligned}
& 2 \alpha_{1}=1+A_{1}-A_{2} A_{5} \\
& \beta_{1}^{2} \equiv\left|x_{1}\right|^{2}-\alpha_{1}^{2}=A_{1}-\alpha_{1}^{2} .
\end{aligned}
$$

Solving (10) for $\beta_{1} \beta_{2}$, squaring for $\beta_{1}^{2} \beta_{2}^{2}$, and substituting from (11) for $\beta_{1}^{2}, \alpha_{1}$, and from the similar relations for $\beta_{2}^{2}, \alpha_{2}$, leads to lengthy algebraic manipulation which yields

$$
\begin{aligned}
A_{1} A_{2} A_{3} A_{4} A_{5}-A_{1}^{2} & A_{3} A_{4}-A_{2}^{2} A_{4} A_{5}-A_{3}^{2} A_{5} A_{1}-A_{4}^{2} A_{1} A_{2}-A_{5}^{2} A_{2} A_{3} \\
& +A_{1} A_{2} A_{4}+A_{2} A_{3} A_{5}+A_{3} A_{4} A_{1}+A_{4} A_{5} A_{2}+A_{5} A_{1} A_{3} \\
& +A_{1} A_{3}+A_{2} A_{4}+A_{3} A_{5}+A_{4} A_{1}+A_{5} A_{2}-A_{1}-A_{2}-A_{3}-A_{4}-A_{5}+1 \equiv 0 .
\end{aligned}
$$

Replacing each $A_{i}$ by $e^{-4 \pi m_{i} / \Gamma_{m}}$ yields the mutual inductance identity.

If the pentagon is regular, the $A_{i}$ are equal, and (12) can be factored:

The allowable roots are

$$
\left(A^{2}-3 A+1\right)^{2}(A+1)=0 .
$$

corresponding to

$$
A=\frac{3 \pm \sqrt{5}}{2}
$$

$$
m=\frac{\Gamma_{m}}{4 \pi} \ln \frac{3+\sqrt{ } 5}{2}
$$

('The two roots are reciprocal, so yield the same absolute value of mutual inductance.)

\section{Application}

In principle, we measure the approximately equal $m_{i}$ by comparison with a standard, $m$. The assumed value of the standard is in error by an unknown factor $(1+\delta)$. The object of the experiment is to determine $\delta$.

If

$$
m_{i}=a_{i} m=\frac{a_{i}}{1+\delta}(1+\delta) m
$$

the apparent, or measured values, are

$$
\hat{m}_{i}=\frac{a_{i} m}{1+\delta}=\frac{m_{i}}{1+\delta}
$$


Substitution into the theorem (12) yields an implicit equation for $\delta$ in terms of the observed $\hat{m}_{i}$.

In practice, there will be random measurement errors associated with each $\hat{m}_{i}$. These will be treated in a later section. A more serious problem is presented by the fact that we cannot measure the inductance per unit length of an infinite cage, but only the change with the length of a finite cage - i.e., end effects must be considered.

\section{End Effects}

Consider two loops of length $l$, the loop planes cutting the transverse plane (fig. 4 ) in the lines $\rho_{i-1}$ and $\rho_{i+1}$.

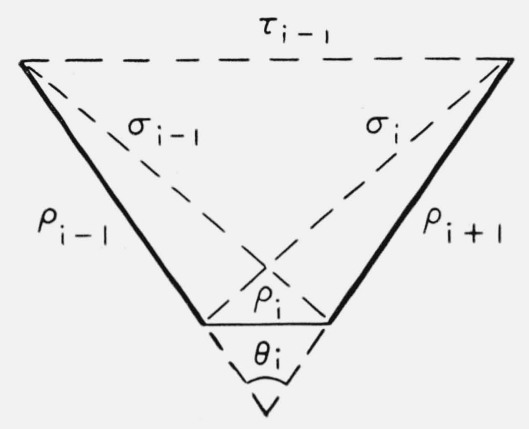

Figure 4

The mutual inductance is given by the double line integral around the two loops:

$$
M_{i}=\frac{\Gamma_{m}}{4 \pi} \oint \oint \frac{d \boldsymbol{\lambda}_{1} \cdot d \boldsymbol{\lambda}_{2}}{R_{12}}
$$

End effects arise from the truncation of the cage at length $l$, and also from the couplings among the shorting bars of length $\rho_{i-1}$ and $\rho_{i+1}$ at the cage ends. The expression for $M_{i}$ becomes:

$$
\begin{aligned}
\frac{4 \pi M_{i}}{\Gamma_{m}}=\int_{0}^{l} \int_{0}^{l} d x d y\left\{\left[(x-y)^{2}+\rho_{i}^{2}\right]^{-1 / 2}+\left[(x-y)^{2}+\tau_{i-1}^{2}\right]^{-1 / 2}-\left[(x-y)^{2}+\sigma_{i-1}^{2}\right]^{-1 / 2}\right. \\
\left.-\left[(x-y)^{2}+\sigma_{i}^{2}\right]^{-1 / 2}\right\}+2 \cos \theta_{i} \int_{0}^{\rho_{i-1}} \int_{0}^{\rho_{i+1}}\left\{\frac{1}{r}-\frac{1}{\sqrt{l^{2}+r^{2}}}\right\} d u d v
\end{aligned}
$$

where $r$ is the distance in the transverse plane between point $u$ on $\rho_{i-1}$ and point $v$ on $\rho_{i+1}$.

Since we are considering inductance per unit length, we are interested in

$$
m_{i} \equiv \frac{\Delta M_{i}}{\Delta l} \doteq \frac{\partial M_{i}}{\partial l}+\frac{\Delta l}{2} \frac{\partial^{2} M_{i}}{\partial l^{2}}
$$

particularly when $l$ is large compared to the cross dimensions of the cage. We have

$$
\begin{aligned}
& \frac{4 \pi}{\Gamma_{m}} \frac{\partial M_{i}}{\partial l}=2 \int_{0}^{l} d x\left\{\left[(l-x)^{2}+\rho_{i}^{2}\right]^{-1 / 2}+\left[(l-x)^{2}+\tau_{i-1}^{2}\right]^{-1 / 2}-\left[(l-x)^{2}+\sigma_{i-1}^{2}\right]^{-1 / 2}\right. \\
&\left.-\left[(l-x)^{2}+\sigma_{i}^{2}\right]^{-1 / 2}\right\}+2 l \cos \theta_{i} \int_{0}^{\rho_{i-1}} \int_{0}^{\rho_{i}+1} d u d v\left(l^{2}+r^{2}\right)^{-3 / 2}=\ln \frac{\sigma_{i-1}^{2} \sigma_{i}^{2}}{\tau_{i-1}^{2} \rho_{i}^{2}} \\
&+2 \ln \frac{\left(l+\sqrt{l^{2}+\rho_{i}^{2}}\right)\left(l+\sqrt{l^{2}+\tau_{i-1}^{2}}\right)}{\left(l+\sqrt{l^{2}+\sigma_{i}^{2}}\right)\left(l+\sqrt{l^{2}+\sigma_{i-1}^{2}}\right)}+2 l \cos \theta_{i} \int_{0}^{\rho_{i-1}} \int_{0}^{\rho_{i+1}} d u d v\left(l^{2}+r^{2}\right)^{-3 / 2} .
\end{aligned}
$$


For large $l$, this is approximated by

$$
\begin{gathered}
\frac{4 \pi}{\Gamma_{m}} \frac{\partial M_{i}}{\partial l} \doteq \ln \frac{\sigma_{i-1}^{2} \sigma_{i}^{2}}{\tau_{i-1}^{2} \rho_{i}^{2}}+\frac{\rho_{i}^{2}+\tau_{i-1}^{2}-\sigma_{i-1}^{2}-\sigma_{i}^{2}}{2 l^{2}}+\frac{2 \rho_{i-1} \rho_{i+1} \cos \theta_{i}}{l^{2}}-\frac{3}{16 l^{4}}\left(\rho_{i}^{4}+\tau_{i-1}^{4}-\sigma_{i-1}^{4}-\sigma_{i}^{4}\right) \\
-\frac{3}{2} \frac{\rho_{i-1} \rho_{i+1} \cos \theta_{i}}{l^{4}}\left\{\tau_{i-1}^{2}+\rho_{i}^{2}+\rho_{i-1} \rho_{i+1} \cos \theta_{i}+\frac{3}{2}\left(\rho_{i-1}^{2}+\rho_{i+1}^{2}\right)\right\}
\end{gathered}
$$

The distances in figure 4 are related by

simplifying (17) to

$$
\rho_{i}^{2}+\tau_{i-1}^{2}-\sigma_{i-1}^{2}-\sigma_{i}^{2}=-2 \rho_{i-1} \rho_{i+1} \cos \theta_{i}
$$

$$
\begin{aligned}
\frac{4 \pi}{\Gamma_{m}} \frac{\partial M_{i}}{\partial l}=\ln \frac{\sigma_{i-1}^{2} \sigma_{i}^{2}}{\tau_{i-1}^{2} \rho_{i}^{2}}+\frac{\rho_{i-1} \rho_{i+1} \cos \theta_{i}}{l^{2}}+ & \frac{1}{l^{4}}\left\{\frac { 3 } { 1 6 } \left(\rho_{i}^{4}+\tau_{i-1}^{4}-\sigma_{i-1}^{4}\right.\right. \\
& \left.\left.-\sigma_{i}^{4}+4 \rho_{i}^{2} \tau_{i-1}^{2}-4 \sigma_{i-1}^{2} \sigma_{i}^{4}\right)-\left(\rho_{i-1}^{2}+\rho_{i+1}^{2}\right) \rho_{i-1} \rho_{i+1} \cos \theta_{i}\right\} .
\end{aligned}
$$

The second derivative, $\partial^{2} M i / \partial l^{2}$, is easily found and leads to

$$
\frac{4 \pi}{\Gamma_{m}} \frac{\Delta M_{i}}{\Delta l} \doteq \ln \frac{\sigma_{i-1}^{2} \sigma_{i}^{2}}{\tau_{i-1}^{2} \rho_{i}^{2}}+\frac{\rho_{i-1} \rho_{i+1} \cos \theta_{i}}{l^{2}}\left(1-\frac{2 \Delta l}{l}\right)+\frac{a}{l^{4}}\left(1-\frac{4 \Delta l}{l}\right)
$$

where $a$ stands for the coefficient of $1 / l^{4}$ in eq (18).

For large $\Delta l$, integration of (18) yields

$$
\frac{4 \pi}{\Gamma_{m}} \frac{M_{i}\left(l_{2}\right)-M_{i}\left(l_{1}\right)}{l_{2}-l_{1}} \doteq \ln \frac{\sigma_{i-1}^{2} \sigma_{i}^{2}}{\tau_{i-1}^{2} \rho_{i}^{2}}+\frac{\rho_{i-1} \rho_{i+1} \cos \theta_{i}}{l_{1} l_{2}}+\frac{a}{3} \frac{l_{1}^{2}+l_{1} l_{2}+l_{2}^{2}}{l_{1}^{3} l_{2}^{3}}
$$

For a regular pentagon, $\sigma^{2}=\tau^{2}=\frac{3+\sqrt{5}}{2} \delta^{2}, \quad \theta=\pi / 5$, yielding

$$
\frac{4 \pi}{\Gamma_{m}} \frac{\partial M}{\partial l} \doteq \ln \frac{3+\sqrt{5}}{2}+\frac{1+\sqrt{5}}{4} \rho^{2} / l^{2}
$$

The $\rho^{2} / l^{2}$ end-effect is intolerable. Equation (19) suggests the use of an octagon, $\cos \theta=0$. For a regular octagon,

yielding

$$
\sigma^{2}=\rho^{2}(2+\sqrt{2}), \quad \tau^{2}=\rho^{2}(3+2 \sqrt{2})
$$

$$
\frac{4 \pi}{\Gamma_{m}} \frac{\partial M}{\partial l} \doteq \ln 2-3 \frac{(3+2 \sqrt{2})}{8} \rho^{4} / l^{4}
$$

with a tolerable end-effect. Errors due to irregularity of the octagon will be treated later.

\section{Octagonal Cage}

Since cross ratios are invariant under bilinear transformations, three points of a polygonal cage can be chosen arbitrarily. This implies that there are $2(n-3)$ independent mutual inductances. For the pentagon, $2(n-3)=4$, providing one identity among the five mutual inductances. In the case of an octagon, there are 10 independent mutual inductances, so the 8 pairs of mutually perpendicular sides are insufficient. There are, however, additional pairs of perpendicular loops (fig. 5). Fortunately, the dotted pair and the solid pair have the same nominal value of mutual inductance. Among the 16 mutual inductances now under consideration, there are two circuit identities, leaving 14 essentially independent measurements. This is a sufficient overdetermination of the cross ratios of the cage to yield a reasonable estimate of the validity of the final determination of $\delta$ in the presence of random errors of measurement. 


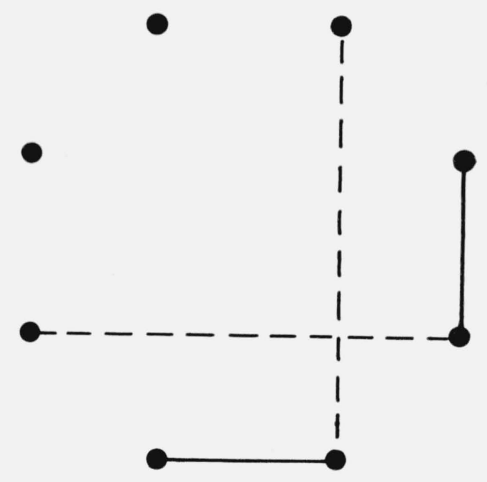

Figure 5

For the two sets of mutual inductances per unit length, of an infinite cage, we have

$$
\begin{aligned}
m_{i} & =\frac{\Gamma_{m}}{4 \pi} \ln \frac{\sigma_{i-1}^{2} \sigma_{i}^{2}}{\tau_{i-1}^{2} \rho_{\imath}^{2}} \\
n_{i} & =\frac{\Gamma_{m}}{4 \pi} \ln \frac{\sigma_{i-2}^{2} \sigma_{i+1}^{2}}{\rho_{i}^{2} \tau_{i+3}^{2}}, \text { indices } \bmod 8 .
\end{aligned}
$$

It does not seem feasible to express the geometric identity among the cross ratios explicitly, as was done for the pentagon eq (12). There is, however, a useful procedure for the application of the inherent identity, i.e., for the determination of the calibration constant, $\delta$. We attribute all random measurements errors to random variations of the standard, i.e., to variations in $\delta$. This artifice simplifies the problem and at the same time yields a conservative estimate of the reliability of the calibration of the standard inductor.

We modify eq (14) to the form

$$
\begin{aligned}
& \hat{m}_{i}=m_{i} /\left(1+\delta_{i}\right) \\
& \hat{n}_{i}=n_{i} /\left(1+\delta_{i+8}\right), \quad i=0 \ldots 7
\end{aligned}
$$

to allow for errors in the 16 inductance determinations. If there were no experimental errors, all $\delta_{i}$ would be equal, and would be overdetermined by the 16 eq (24). A computer program could be developed to assign positions to the cage wires that would make eq (24) compatible, thus yielding both $m_{i}$ and $\delta$. In the presence of errors, our best estimate of $\delta$ arises from assigning cage coordinates to minimize the variance of the resulting $\delta_{i}{ }^{2}$

$$
\begin{aligned}
\varphi & =\frac{1}{16} \sum_{0}^{15}\left(\delta_{i}-\delta\right)^{2} \\
\varphi_{\min } & =s^{2}=\bar{\delta}_{i}^{2}-\bar{\delta}_{i}^{2}, \quad \delta=\bar{\delta}_{i} .
\end{aligned}
$$

Cage coordinates are to be assigned to minimize $s^{2}$. From eq (24), we have

$$
s^{2}=\frac{1}{16} \sum_{0}^{7}\left(\frac{m_{i}-\hat{m}_{i}}{\hat{m}_{i}}\right)^{2}+\frac{1}{16} \sum_{0}^{7}\left(\frac{n_{i}-\hat{n}_{i}}{\hat{n}_{i}}\right)^{2}-\left\{\frac{1}{16} \sum_{0}^{7} \frac{m_{i}-\hat{m}_{i}}{\hat{m}_{i}}+\frac{1}{16} \sum_{0}^{7} \frac{n_{i}-\hat{n}_{i}}{\hat{n}_{i}}\right\}^{2} .
$$


"The theoretical values $m_{i}, n_{i}$, are computed from assigned cage coordinates using eq (23). Since the $m_{i}$ and $n_{i}$ depend only upon cross ratios, and cross ratios are unaffected by bilinear transformations, three of the cage points can be arbitrarily assigned. The other five are then adjusted to minimize $s^{2}$. When $s^{2}$ is minimized, the term in braces in (26) is the desired value of $\delta$. The measured values $\hat{m}_{i}$ and $\hat{n}_{i}$ will be essentially equal, so this term can be approximated by

$$
\delta \doteq \frac{\Sigma m_{i}+\Sigma n_{i}}{\Sigma \hat{m}_{i}+\Sigma \hat{n}_{i}}-1
$$

For displacement of cage wires from the vertices of a regular octagon, both $\Sigma m_{i}$ and $\Sigma n_{i}$ are at a saddle point. In fact, for displacement of any one wire in a direction making an angle $\varphi$ with the radius from the octagon center, we have

$$
\begin{aligned}
& \frac{\partial \sum_{i} m_{i}}{\partial(r / \rho)}=\frac{\partial \sum_{i} n_{i}}{\partial(r / \rho)}=0 \\
& \frac{\partial^{2} \sum_{i} m_{i}}{\partial(r / \rho)^{2}}=\frac{\partial^{2} \sum_{i} n_{i}}{\partial^{2}(r / \rho)^{2}}=-\frac{\Gamma_{m}}{\pi}(2-\sqrt{2}) \cos 2 \varphi .
\end{aligned}
$$

Thus an ignored displacement of one wire from its regular position would affect $s^{2}$, but not $\delta$, to first order. The second order effect on $\delta$ vanishes if averaged over all directions of displacement.

\section{End Effects}

The use of a regular octagon reduces end effects to terms in $1 / l^{4}$. Departures from regularity introduce end effects in $1 / l^{2}$. Formulas (18) and (19) in $M_{i}$ are converted to the corresponding formulas in $N_{i}$ by the following changes:

$$
\begin{array}{lll}
\sigma_{i-1} \rightarrow \sigma_{i-2} & \rho_{i} \rightarrow \rho_{i} & \theta_{i} \rightarrow \varphi_{i} \\
\sigma_{i} \rightarrow \sigma_{i+1} & \rho_{i-1} \rightarrow \tau_{i-2} & \\
\tau_{i-1} \rightarrow \tau_{i+3} & \rho_{i+1} \rightarrow \tau_{i} &
\end{array}
$$

where $\varphi_{i}$ is the angle between $\tau_{i-2}$ and $\tau_{i}$ (fig. 6).

The computed $m_{i}$ and $n_{i}$ used for minimizing $s^{2}$ eq (26) can be taken from eq (19) and its $n_{i}$ equivalent, thus automatically allowing for end effects. Ignoring these end effects by using the infinite-cage formula in the minimization would still produce only a small error.

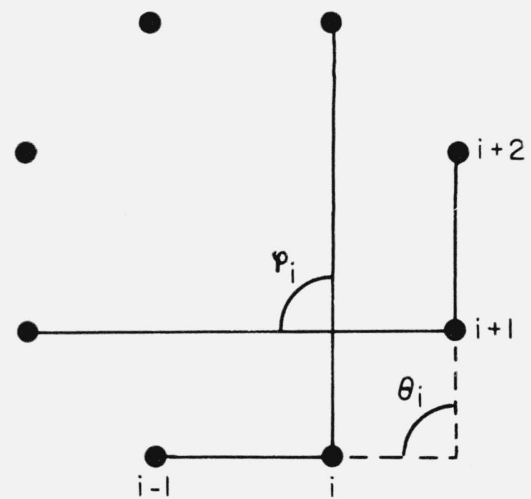

Figure 6 
For a regular octagon, eq (21) yields a fractional error of

$$
-\frac{3(3+2 \sqrt{2})}{8 \ln 2} \rho^{4} / l^{4}
$$

in $m_{i}$, and the corresponding formula for $n_{i}$ yields a fractional error of

$$
-\frac{3(3+2 \sqrt{2})^{3}}{8 \ln 2} \rho^{4} / l^{4}
$$

The resulting fractional error in $\Sigma\left(m_{i}+n_{i}\right)$ is approximately $-7 \rho^{4} / l^{4}$, which is of the order of $10^{-7}$ for $\rho / l=0.01$.

We must also investigate the error arising from the $1 / l^{2}$ term for a slightly irregular octagon. For the $m_{i}$, we have

$$
\epsilon_{i}=\rho_{i-1} \rho_{i+1} \cos \theta_{i} / l^{2} \ln 2
$$

and for the $n_{i}, \epsilon_{i}=\tau_{i-2} \tau_{i} \cos \phi_{i} / l^{2} \ln 2$. By eq (27), these terms lead to an erroneous $\delta$, or fractional error in the mutual inductance assigned to the standard, equal to $\bar{\epsilon}_{i}$ :

$$
\delta_{e}=\frac{\Sigma \boldsymbol{\epsilon}_{i}}{16}=\frac{\Sigma\left(\boldsymbol{\rho}_{i-1} \cdot \boldsymbol{\rho}_{i+1}+\boldsymbol{\tau}_{i-2} \cdot \boldsymbol{\tau}_{i}\right)}{16 l^{2} \ln 2} .
$$

If points 1, 2, 3 are fixed, and the other five displaced from nominal by $\boldsymbol{\eta}_{i}$, we find

If

$$
\Sigma \boldsymbol{\epsilon}_{i}=\frac{2}{l^{2} \ln 2}\left[2 \boldsymbol{\eta}_{5} \cdot \boldsymbol{\eta}_{7}+2 \boldsymbol{\eta}_{6} \cdot \boldsymbol{\eta}_{8}+\boldsymbol{\eta}_{4} \cdot \boldsymbol{\eta}_{6}-\boldsymbol{\eta}_{4} \cdot \boldsymbol{\eta}_{5}-\boldsymbol{\eta}_{4} \cdot \boldsymbol{\eta}_{7}-\boldsymbol{\eta}_{5} \cdot \boldsymbol{\eta}_{6}-\boldsymbol{\eta}_{5} \cdot \boldsymbol{\eta}_{8}-\boldsymbol{\eta}_{6} \cdot \boldsymbol{\eta}_{7}-\boldsymbol{\eta}_{7} \cdot \boldsymbol{\eta}_{8}\right] .
$$

$$
|\boldsymbol{\eta}| \leq \alpha \text {, then }\left|\Sigma \epsilon_{i}\right| \leq \frac{2}{l^{2} \ln 2} 11 \alpha^{2}
$$

and

For

$$
\left|\delta_{e}\right| \leq 2 \alpha^{2} / l^{2}
$$

$$
\alpha \sim 0.02 \rho \text { and } \rho \sim 0.01 l,\left|\delta_{e}\right| \leq 10^{-7} \text {. }
$$

\section{Other Errors}

A bent wire, with zero average displacement, is expected to yield an error similar to that of an ignored displacement, but of lower order. An ignored displacement affects $s^{2}(\delta)$ but not $\bar{\delta}$. This would have more effect on the reliability of calibration than on the calibration constant itself. Exact analysis of the bent wire situation is difficult; the effect should be checked experimentally by adding known perturbations to the cage.

The analysis has assumed ideal wires. Actual construction requires finite diameters. It is well known (and easily shown) that at zero frequency, such wires are equivalent to ideal wires. Proximity effects on the current distribution can be experimentally lumped with skin effect, and the net effect evaluated by making measurements at various frequencies. Nongeometrical effects, such as produced by the permeability of the wire and supports, will be investigated in the future.

\section{Conclusion}

It has been shown that there is a mutual inductance analog of the Thompson-Lampard theorem. In particular, a design is proposed wherein end effects are substantially reduced. The mutual inductance of this design is $\ln 2 / 10 \mu \mathrm{h} / \mathrm{m}$.

(Paper 67B1-91) 